\title{
$Z \alpha$-expansion for self-energy radiative corrections to parity nonconservation in atoms
}

\author{
M.Yu.Kuchiev * \\ School of Physics, University of New South Wales,Sydney 2052, Australia
}

\begin{abstract}
The self-energy and vertex QED radiative corrections to the parity nonconservation (PNC) amplitude in atoms are obtained using the perturbation theory in powers of $\alpha Z$. The calculated linear in $\alpha Z$ term gives $-0.6 \%$ for the PNC amplitude in Cs. The estimated nonlinear terms make corrections larger $-0.9(2) \%$. This result brings the experimental data for the $6 s-7 s$ transition in ${ }^{133} \mathrm{Cs}$ in agreement with the standard model.
\end{abstract}

32.80.Ys, 11.30.Er, 31.30.Jv

Typeset using REVTEX

*kuchiev@newt.phys.unsw.edu.au 
This work is inspired by a recently discovered pronounced deviation of experimental data on parity nonconservation (PNC) in atoms from predictions of the standard model. The deviation was found in the $6 s-7 s$ PNC amplitude in ${ }^{133} \mathrm{Cs}$, that was investigated by Bouchiat and Bouchiat [1], Gilbert and Wieman [2], and, most recently with the highest precision $0.3 \%$, by Wood et al [3]. The experimental progress was matched by atomic PNC calculations that are crucial for the analysis of the experimental data. Accurate calculations of Refs. [4, [0] have been revisited recently by Kozlov et al [6] and Dzuba et al [7:8]. Bennett and Wieman [9] compared the theoretical data [4.5] with available experimental data on dipole amplitudes, polarizabilities and hyperfine constants for $\mathrm{Cs}$, and suggested that the theoretical error for the PNC amplitude should be reduced from $1 \%$ to $0.4 \%$. This suggestion is supported by Ref. [8] that estimates the theoretical error as $0.5 \%$. There have been discovered recently several, previously neglected phenomena that contribute at this level of accuracy. The Breit corrections give $-0.6 \%$ as was found by Derevianko [10 and confirmed in [11,6]. Sushkov [12 proposed that the radiative corrections may be important. This suggestion is supported by the calculations of Johnson et al [13] who demonstrated that the QED vacuum polarization gives $0.4 \%$, the value confirmed in [14.7.15]. Ref. [9] indicates that there is a $2.3 \sigma$ deviation of the weak charge $Q_{\mathrm{W}}$ extracted from the atomic PNC amplitude [3] from predictions of the standard model [28]. More recent analyses [13],8], in which the Breit corrections $(-0.6 \%)$ and the QED vacuum polarization $(0.4 \%)$ were included, give similar deviations $2.2 \sigma$ and $2.0 \sigma$ respectively.

We evaluate the self-energy and vertex radiative corrections, called the e-line corrections below. They were considered previously by Marciano and Sirlin [16] and Lynn and Sandars [17] using the plane wave approximation that resulted in a small value $\sim 0.1 \%$. An attempt to take into account the strong Coulomb field in this problem was made in Ref. [14] that concluded that the e-line corrections are negligible because they are not enhanced by large logarithmic factors (which play an important role for the vacuum polarization, as was demonstrated by Milstein and Sushkov in the cited paper and confirmed in Ref. [15]). We show that the strong Coulomb field makes the e-line corrections large due to unexpected numerical interplay. Our results are in qualitative agreement with conclusions of recent Ref. [18] that demonstrated for the first time that the e-line corrections are important. We find quantitative agreement with this paper as well, the correction $-0.9 \%$ obtained for the $6 s-7 s$ PNC amplitude in ${ }^{133} \mathrm{Cs}$ in the present paper matches the result $-0.7(2) \%$ of 18 . This correction brings the experimental data of Wood et al [3] in agreement with the standard model. The present paper relies on the perturbation theory in powers of $\alpha Z$. It should be noted that [18] uses very different approach, based on an identity that expresses the radiative corrections to the PNC amplitude via corrections to the energy shifts due to the finite nuclear size that have been calculated previously in Refs. [20 23].

Diagrams (1),(2) and (3) in Fig.1 describe the e-line radiative corrections to the PNC amplitude in the first order of the perturbation theory in $\alpha Z$. The weak PNC interaction between the electron and the nucleus originates from the Z-boson exchange. It is described by the Hamiltonian (relativistic units $\hbar=c=m=1$ are used, if not stated otherwise)

$$
H_{\mathrm{PNC}}=(2 \sqrt{2})^{-1} G_{F} Q_{\mathrm{W}} \rho(r) \gamma_{5},
$$

where $G_{F}$ is the Fermi constant, $Q_{W}$ is the nuclear weak charge, and the nuclear density for our purposes can be approximated by the delta function, $\rho(r)=Z \delta(\mathbf{r})$. The perturbation 
induced by the Hamiltonian (西) is described by a factor $G_{F} Q_{\mathrm{W}} Z /(2 \sqrt{2}) \gamma_{0} \gamma_{5}$ that appears for all the diagrams considered. Since we are interested merely in a relative contribution of the corrections, the constant $G_{F} Q_{\mathrm{W}} Z /(2 \sqrt{2})$ will be dropped in all formulas below to simplify notation. We use the Feynman gauge for the photon propagator $D_{\mu \nu}(q)=4 \pi g_{\mu \nu} /\left(q^{2}-\lambda^{2}\right)$, and introduce the density matrix $\wp=(1 / 4)(\hat{p}+1)\left(1+\hat{a} \gamma_{5}\right)$ that distinguishes the electron state with the given momentum $p_{\mu}$ and the polarization four-vector $a_{\mu}$. The latter can be expressed via the polarization three-vector $\boldsymbol{\zeta}, a_{0}=\mathbf{p} \cdot \boldsymbol{\zeta}, \mathbf{a}=\boldsymbol{\zeta}+\mathbf{p}(\mathbf{p} \cdot \boldsymbol{\zeta}) /\left(1+p_{0}\right)$. It suffices to discuss the forward scattering in which the electron momentum $p_{\mu}$ and its polarization $a_{\mu}$ remain intact, and restrict our consideration to the limit $\mathbf{p} \rightarrow 0$ because all important events that contribute to the process happen at separations $r \simeq 1 / m$ that are much less than the atomic radius. The only pseudoscalar available in this kinematics is $a_{0}=\mathbf{p} \cdot \boldsymbol{\zeta}$ that should appear as a common factor for all diagrams for the PNC amplitude. We need therefore to extract the lowest, linear in $\mathbf{p}$ term of the $\mathbf{p} \rightarrow 0$ expansion. The analytical expression for each Feynman diagram can be written in the following form

$$
-i \alpha \int \frac{d^{4} q}{(2 \pi)^{4}} \frac{d^{3} k}{(2 \pi)^{3}} \frac{4 \pi}{q^{2}-\lambda^{2}} \frac{-4 \pi Z \alpha}{\mathbf{k}^{2}} \frac{\operatorname{Tr}(\mathcal{N} \wp)}{\mathcal{D}}
$$

where $q$ is the photon momentum, $\mathbf{k}$ is the momentum running over the Coulomb and the PNC weak interactions, $\mathrm{Tr}$ is the trace over the spinor indexes, and the nominator $\mathcal{N}$ and denominator $\mathcal{D}$ are specific for each diagram. To clarify notation let us present explicitly the nominators $\mathcal{N}_{1}, \mathcal{N}_{2}$ for one Feynman diagram of the type (1) and one diagram of the type (2) in Fig.1

$$
\begin{aligned}
& \mathcal{N}_{1}=\gamma^{\mu}(\hat{P}+1) \gamma_{0}(\hat{Q}+1) \gamma_{\mu}(\hat{R}+1) \gamma_{0} \gamma_{5}, \\
& \mathcal{N}_{2}=\gamma^{\mu}(\hat{P}+1) \gamma_{0}(\hat{Q}+1) \gamma_{0} \gamma_{5}(\hat{P}+1) \gamma_{\mu},
\end{aligned}
$$

where $P=p+q, Q=p+k+q, R=p+k, k=(0, \mathbf{k})$. The vertexes $\gamma_{0}$ and $\gamma_{0} \gamma_{5}$ in Eq.(3) originate from the Coulomb and the PNC weak interaction respectively. Using Eqs. (A1) we find from Eq.(3) (and similar expressions for the Feynman diagrams that are topologically identical to either diagram (1), or (2) in Fig.1)

$$
\begin{aligned}
& \sum \operatorname{Tr}\left(\mathcal{N}_{1} \wp\right)=-8\left[2 P_{0}\left(a_{0}+(p R) a_{0}-(a R) p_{0}\right)-(Q \tilde{P} \tilde{R} a)\right] \\
& \sum \operatorname{Tr}\left(\mathcal{N}_{2} \wp\right)=-4[(\tilde{Q} a)-(P \tilde{Q} \tilde{R} a)] .
\end{aligned}
$$

Here $(x y)$ is a scalar product of vectors, $(x y) \equiv x_{\mu} y^{\mu}$, and the symbol $(x y z u)$ stands for the following invariant of four four-vectors $(x y z u) \equiv(x y)(z u)-(x z)(y u)+(x u)(y z)$. The tilde sign in Eq.(4) marks the inversion of a four-vector $\tilde{x}^{\mu} \equiv\left(x_{0},-\mathbf{x}\right)$. Summation in (4) and (5) includes all Feynman diagrams topologically identical to diagram (1) and (2) in Fig.1 respectively. The conventional parameterization permits the denominator for diagram (1) in Fig. 1 to be presented in the form

$$
\begin{array}{r}
\frac{1}{\left(q^{2}-\lambda^{2}\right) \mathcal{D}_{1}}=\frac{2}{R^{2}-1} \int_{0}^{1} \frac{1}{D_{1}^{3}} \delta\left(\sum_{i=1}^{3} \xi_{i}-1\right) \prod_{i=1}^{3} d \xi_{i} \\
D_{1}=\xi_{1}\left(P^{2}-1\right)+\xi_{2}\left(Q^{2}-1\right)+\xi_{3}\left(q^{2}-\lambda^{2}\right) .
\end{array}
$$


Using similar presentation for diagram (2) we calculate the integral over the photon momentum $q$ in Eq.(2) for these two diagrams with the help of Eqs.(A2). Expanding the found results up to the first power in $\mathbf{p}$ we integrate them over the angular variables $\Omega_{\mathbf{k}}$ of the momentum $\mathbf{k}$. These straightforward calculations lead to the following representation for the e-line radiative correction $\delta_{\mathrm{e}-\text { line }}^{\mathrm{PNC}}$ to the $\mathrm{PNC}$ amplitude

$$
\begin{aligned}
& \delta_{\mathrm{e}-\text { line }}^{\mathrm{PNC}}=\mathcal{C}^{\mathrm{PNC}} \alpha^{2} Z \\
& \mathcal{C}^{\mathrm{PNC}}=-\frac{8}{3 \pi^{2}} \int_{0}^{\infty}\left(f_{1}\left(k^{2}\right)+f_{2}\left(k^{2}\right)+f_{3}\left(k^{2}\right)\right) d k
\end{aligned}
$$

Note that $\delta_{\mathrm{e}-\text { line }}^{\mathrm{PNC}}$ is a relative correction, i.e. the correction divided by the main amplitude (which in our notation is equal to $\left(-a_{0}\right)$ ). The integrand in Eq.(8) includes contributions from all diagrams, each $f_{i}(z), z=k^{2}$ originates from the (i)-th diagram in Fig.1. The above described procedure presents the functions $f_{1}(z), f_{2}(z)$ via the two-dimensional integrals

$$
\begin{aligned}
& f_{1}(z)=\frac{1}{z} \int_{0}^{1} d u \int_{0}^{u} d v\left(\frac{A}{D}-\frac{B}{D^{2}} z+\frac{C}{u^{2}}+2 \ln \frac{D}{u^{2}}\right) \\
& f_{2}(z)=\int_{0}^{1} d u \int_{0}^{u} d v(u-v)\left(\frac{E}{2 D^{2}}-\frac{F}{D^{3}} z-\frac{4}{u^{3} z}-\frac{3(1-u)}{2 D}\right)
\end{aligned}
$$

where the symbols $A, B, \ldots F$ in the integrands are defined as follows

$$
\begin{aligned}
& A=(1-u)(4-u+2 v)+((1-u)(2-v)+2 v(1-v)) z \\
& B=(1-u) v((1-u)(2+u-2 v)+v(1-v) z) \\
& C=4-(1-u)(4-u+2 v) \\
& D=u^{2}+v(1-v) z \\
& E=(1-u)\left[3\left(1+(1-u)^{2}\right)+v(1-v) z\right] \\
& F=(1-u) v\left[(1-u)^{2}(1-3 v)+1-v+v^{2}(1-v) z\right] .
\end{aligned}
$$

The integration over $u, v$ in (9), (10) arises from the integral in Eq.(6) (and a similar expression for the diagram (2)), $u=\xi_{1}+\xi_{2}, v=\xi_{2}$. Diagram (3) in Fig.1 is expressed directly via the mass operator calculated by Feynman [27], resulting in

$$
\begin{aligned}
f_{3}(z) & =-\frac{1}{2 z}\left(2 g(z)+z g^{\prime}(z)\right) \\
g(z) & =\frac{1}{2(1-z)}\left(2-z+\frac{z^{2}+4 z-4}{1-z} \ln z\right)+1 .
\end{aligned}
$$

Eqs.(9),(10), and (12) are derived using conventional renormalization procedure to deal with the logarithmic ultraviolet divergences. In the Feynman gauge each one of the diagrams in Fig. 1 possesses also the infrared singularity $\propto \ln \lambda, \lambda \rightarrow 0$. It is well known that for problems of the type considered the infrared divergence should not manifest itself, and, indeed, we find that it cancels out for the sum of all diagrams. This cancellation allows us to derive convergent integral representations (9),(10), and to express (12) in terms of a well defined, finite function $g(z)(13)$. Since the functions $A, B, \ldots F$ in Eq.(11) are all polynomials, a 
large number of, probably all, integrations in (9),(10) can be carried out in an analytical form. However, our main goal here is a numerical value for the factor $\mathcal{C}^{\mathrm{PNC}}$. We rely, therefore, on numerical calculations whenever it is more convenient. Calculating integrals in Eqs.(9),(10) we find the functions $f_{1}(z)$ and $f_{2}(z)$. I Combining them with $f_{3}(z)$ from (12) we find the function $f(z)=\sum_{i=1}^{3} f_{i}(z)$ in the integrand in Eq.(8). Its asymptotes are

$$
f(z)=\frac{4}{3} \times\left\{\begin{array}{cl}
-\ln z+\frac{1}{3}, & z \rightarrow 0 \\
(\ln z+\text { const }) \frac{1}{z}, & z \rightarrow \infty .
\end{array}\right.
$$

Finally, calculating with the found function the integral in Eq.(8) we derive the e-line radiative correction (7)

$$
\delta_{\mathrm{e}-\text { line }}^{\mathrm{PNC}}=-1.97 \alpha^{2} Z
$$

For heavy atoms the found correction is negative and large. This qualitative result agrees with Ref. [18]. Moreover, numerical results for the Cs atom are also in good agreement. Eq.(15) predicts that the e-line correction is $-0.6 \%$, which is close to $-0.7(2) \%$ found in 18 .

Eq.(15) shows that the e-line correction is large due to a large coefficient $\sim 2.0$ in its righthand side. Naively one could expect this coefficient to be smaller, of the order of $\sim 1 / \pi$. It is interesting that similar "numerical enhancement" happens for the e-line radiative correction for the energy shift that is due to the finite nuclear size (FNS). Analytically this correction was examined by Pachucki [24] and Eides and Grotch [25]. In our notation their result can be written as

$$
\delta_{\mathrm{e}-\text { line }}^{\mathrm{FNS}}=\mathcal{C}^{\mathrm{FNS}} \alpha^{2} Z=2.978 \alpha^{2} Z
$$

where $\mathcal{C}^{\text {FNS }}=(3 / 2) 1.985=2.978$, the coefficient 1.985 is taken from Eq.(9) of [25]. Eq.(16]) shows, that, indeed, the e-line corrections to the FNS energy shift are governed by the large coefficient $\sim 3.0$, similar to Eq.(15) for the corrections to the PNC amplitude. Fig.2 examines this similarity in more detail. It shows data available for relative e-line corrections for the two problems mentioned above, namely for the PNC amplitude and FNS energy shift. The linear in $Z$ approximations (15),(16) (that are valid for sufficiently small values of $Z$ ) are compared in this figure with results of numerical calculations available for large $Z$. The e-line FNS corrections were calculated in Refs. [20 23]. Specifically, the results for FNS shown in Fig.2 were extracted in [18] from [22] (using also Ref. 26] for $Z=55$ ), see details in [18]. The data shown for the PNC amplitude are taken from the same paper [18] that explains that the correction to the PNC amplitude is equal to the average of the corrections to the FNS energy shifts for $S_{1 / 2}$ and $P_{1 / 2}$ levels. The latter ones (i.e. the corrections to the FNS energy shifts of $P_{1 / 2}$ levels) were also calculated in Refs. 20 23]. The results for the PNC amplitude shown in Fig.2 are based on data of [22] (and [26]), see [18] for details.

\footnotetext{
${ }^{1} \mathrm{~A}$ simple way provides an expansion of $1 / D$ in Eqs.(9), (10) in powers of $\left[z v^{2} /\left(u^{2}+v z\right)\right]$. For each term in this series Mathematica [19] reliably performs integrations in (9),(10) in an analytical form. The procedure rapidly converges.
} 
We observe very close quantitative similarity between corrections to PNC and FNS. In both cases the linear approximations (15) and (16) predict large negative corrections, which agrees with results based on numerical calculations for heavy atoms. Numerical validity of the linear approximations seem to be limited by the region below $Z=55$, for higher $Z$ they underestimate the effect. The Cs atom lies on the border, where results of small- $Z$ and large- $Z$ approaches agree reasonably well.

Numerical data used in Ref. [18] incorporates an error that increases for smaller values of $Z$, see Tables III and IV of [22]. In order to reduce an impact of this error we can combine Eq.(15) of the present paper with results of [18]. Let us approximate the nonlinear terms omitted in Eq.(15) by a simplest quadratic term and choose a corresponding coefficient to reproduce the results based on numerical data for very large $Z, Z \sim 90$, where the numerical errors are small. This gives us the following interpolating formula for the corrections to the PNC amplitude

$$
\delta_{\mathrm{e}-\text { line, int }}^{\mathrm{PNC}}=-1.97 \alpha^{2} Z(1+1.55 \alpha Z)
$$

Fig.2 shows that any reasonable interpolation between data available for large-Z and small-Z regions would produce a similar pattern. We conclude therefore that Eq.(17) should give the most reliable numerical data. For the Cs atom we find from it the correction $-0.9(2) \%$, where we adopt the error $0.2 \%$ of [18 (compare this result with $-0.6 \%$ of Eq.(15) and $-0.7(2) \%$ of Ref. [18] mentioned above).

The standard model value for the nuclear weak charge for Cs [28] is

$$
Q_{W}\left({ }^{133} \mathrm{Cs}\right)=-73.09 \pm(0.03) \text {. }
$$

Ref. [8] refined previous calculations of Ref. [4 extracting from the experimental PNC amplitude of Ref. [3] the weak charge

$$
Q_{W}\left({ }^{133} \mathrm{Cs}\right)=-72.18 \pm(0.29)_{\operatorname{expt}} \pm(0.36)_{\text {theor }}
$$

with the theoretical error $0.5 \%$. It is consistent with $Q_{W}\left({ }^{133} \mathrm{Cs}\right)=-72.21 \pm(0.28)_{\operatorname{expt}} \pm$ $(0.34)_{\text {theor }}$ that was adopted in [13] by taking the average of the results of Refs. [4- 6], and accepting the theoretical error $0.4 \%$ of [9]. The weak charge in Eq.(19) deviates from the standard model (18) by $2.0 \sigma$. Taking from Eq.(17) the value $-0.9(2) \%$ for the e-line radiative correction, we derive from Eq.(19)

$$
Q_{W}\left({ }^{133} \mathrm{Cs}\right)=-72.83 \pm(0.29)_{\operatorname{expt}} \pm(0.39)_{\text {theor }}
$$

which brings the experimental results of [3] within the limits of the standard model (18). (Note that even the smaller value for this correction $-0.6 \%$ that follows from the linear approximation Eq.(15) justifies the latter conclusion.)

The calculations presented show that the QED self-energy and vertex radiative corrections bring the experimental results of Wood et al [3] within the limits of the standard model.

This work was supported by the Australian Research Council. I wish to thank Victor Flambaum for numerous discussions, and K.T.Cheng for Ref. 26]. 


\section{APPENDIX A:}

The calculations performed use the following algebraic relations

$$
\begin{aligned}
& \left\langle\gamma_{5}\right\rangle=0, \quad\left\langle\gamma_{\mu} \gamma_{5}\right\rangle=-a_{\mu}, \quad\left\langle\gamma_{\mu} \gamma_{\nu} \gamma_{5}\right\rangle=-\left(p_{\mu} a_{\nu}-p_{\nu} a_{\mu}\right) \\
& \left\langle\gamma_{\mu} \gamma_{\nu} \gamma_{\lambda} \gamma_{5}\right\rangle=-\left(a_{\mu} g_{\nu \lambda}-a_{\nu} g_{\mu \lambda}+a_{\lambda} g_{\mu \nu}\right)-i \epsilon_{\mu \nu \lambda \rho} a^{\rho}
\end{aligned}
$$

where the $\langle X\rangle \equiv \operatorname{Tr}(X \wp)$, and conventional integrals

$$
\begin{aligned}
& \int \frac{d^{4} q}{\left(q^{2}+2 q s-M^{2}\right)^{n}}=\frac{(-1)^{n} i \pi^{2} c_{n}}{\left(M^{2}+s^{2}\right)^{n-2}}, \quad n>2, \\
& \int \frac{q^{\mu} d^{4} q}{\left(q^{2}+2 q s-M^{2}\right)^{n}}=\frac{(-1)^{n-1} i \pi^{2} c_{n} s^{\mu}}{\left(M^{2}+s^{2}\right)^{n-2}}, \quad n>2, \\
& \int \frac{q^{\mu} q^{\nu} d^{4} q}{\left(q^{2}+2 q s-M^{2}\right)^{3}}=\frac{-i \pi^{2}}{2}\left[\frac{s^{\mu} s^{\nu}}{M^{2}+s^{2}}-\frac{1}{2} g^{\mu \nu}\left(\ln \frac{\Lambda^{2}}{M^{2}+s^{2}}-\frac{3}{2}\right)\right], \\
& \int \frac{q^{\mu} q^{\nu} q^{\lambda} d^{4} q}{\left(q^{2}+2 q s-M^{2}\right)^{n}}=\frac{(-1)^{n} i \pi^{2} c_{n}}{\left(M^{2}+s^{2}\right)^{n-3}}\left[-\frac{s^{\mu} s^{\nu} s^{\lambda}}{M^{2}+s^{2}}+\frac{g^{\mu \nu} s^{\lambda}+g^{\mu \lambda} s^{\nu}+g^{\nu \lambda} s^{\mu}}{2(n-3)}\right], n>3,
\end{aligned}
$$

where $c_{n}=1 /[(n-1)(n-2)], M$ is a scalar, $s$ is a four-vector, $q s \equiv(q s)$, and $\Lambda$ is the ultraviolet cut-off. 


\section{REFERENCES}

[1] M.A.Bouchiat and C.Bouchiat J.Phys. (Paris) 35, 899 (1974); 36, 493 (1974).

[2] S.L.Gilbert and C.E.Wieman, Phys.Rev. A 34, 792 (1986).

[3] C.S.Wood, S.C.Bennett, D.Cho, B.P.Masterson, J.L.Roberts, C.E.Tanner, and C.E.Wieman, Science 275, 1759 (1997).

[4] V.A.Dzuba, V.V.Flambaum, and O.P.Sushkov, Phys. Lett A 141, 147 (1989).

[5] S.A.Blundell, J.Sapirstein, and W.R.Johnson, Phys. Rev. D 45, 1602 (1992).

[6] M.G.Kozlov, S.G.Porsev, and I.I.Tupitsyn, Phys. Rev. Lett. 86, 3260 (2001).

[7] V.A.Dzuba, V.V.Flambaum, and J.S.M. Ginges, hep-ph/0111019 (2001).

[8] V.A.Dzuba, V.V.Flambaum, and J.S.M. Ginges, hep-ph/0204134 (2002).

[9] S.C.Bennett and C.E.Wieman, Phys. Rev. Lett. 82, 2484 (1999); 82, 4153 (1999); 83, 889 (1999).

[10] A.Derevianko, Phys. Rev. Lett. 85, 1618 (2000).

[11] V.A.Dzuba, C.Harabati, W.R.Johnson, and M.S.Safronova, Phys. Rev A 63, 044103 (2001).

[12] O.P.Sushkov, Phys. Rev. A, 63, 042504 (2001).

[13] W.R.Johnson, I.Bednyakov, and G.Soff, Phys. Rev. Lett 87, 233001-1 (2001).

[14] A.I.Milstein and O.P.Sushkov, hep-ph/0109257 (2001).

[15] M.Yu.Kuchiev and V.V.Flambaum, hep-ph/0205012 (2002).

[16] W.J.Marciano and A.Sirlin, Phys. Rev. D 27, 552 (1983).

[17] B.W.Lynn and P.G.H.Sandars, J. Phys. B 27, 1469 (1994).

[18] M.Yu.Kuchiev and V.V.Flambaum, hep-ph/0206124 (2002).

[19] S.Wolfram, Mathematica. A system for doing mathematics by computer. (Reading, Mass. Addison-Wesley Pub. 1991).

[20] W.R.Johnson and G.Soff, At. Data Nuc. Data Tables 33, 405 (1985).

[21] S.A.Blundell, Phys. Rev. A 46, 3762 (1992).

[22] K.T.Cheng, W.R.Johnson and J.Sapirstein, Phys. Rev A 47, 1817 (1993).

[23] I.Lindgren, H.Persson, S.Salomonson, and A.Ynnerman, Phys. Rev. A 47, 4555 (1993).

[24] K.Pachucki, Phys. Rev. A 48, 120 (1993).

[25] M.I.Eides and H.Grotch, Phys. Rev. A 56, R2507 (1997).

[26] K.T.Cheng. Private communication (2002).

[27] R.P.Feynman, Phys.Rev. 76, 769 (1949).

[28] D.E.Groom et al, Eur. Phys. J. C 15, 1 (2000). 


\section{FIGURES}

FIG. 1. The QED self-energy and vertex corrections, called e-line corrections in the text, to the PNC matrix element. For each diagram one of the wavy legs shows the Coulomb interaction with the nucleus, another one the weak PNC interaction with the nucleus. Each diagram represents all possible Feynman diagramms with the given topological structure.

FIG. 2. The thin dotted line, thick dotted line, and solid line: e-line corrections to the PNC amplitude that follow from Eq.(15), Ref.[18], and Eq.(17). The thin dashed line and thick dashed line: the e-line corrections to the FNS energy shifts that follow from Eq.(16) (derived

from [25]), and Ref.[18] (based on calculations of [22]), the dashed-dotted line: the interpolation $\delta_{\mathrm{e}-\text { line, int }}^{\mathrm{FNS}}=-2.978 \alpha^{2} Z(1+0.85 \alpha Z)$ between the two latter lines. 


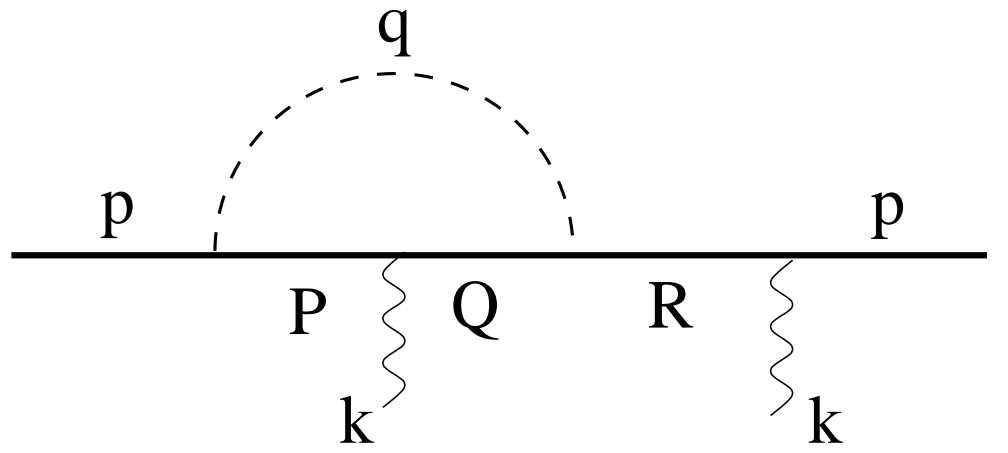

(1)

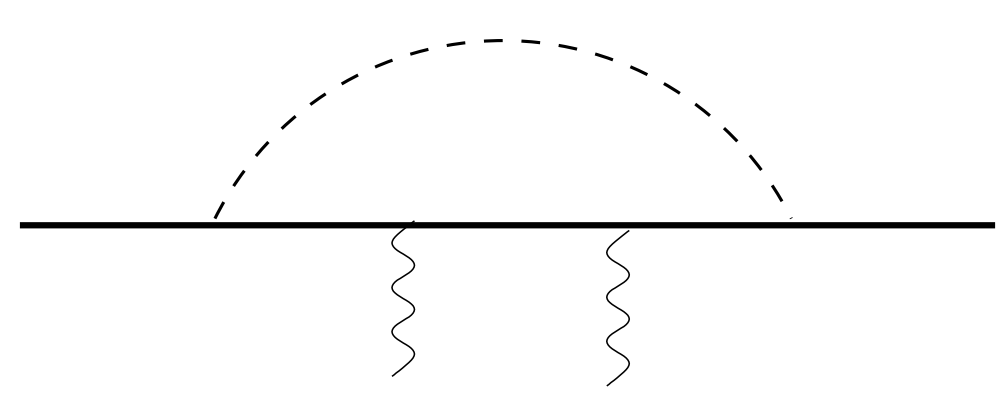

(2)

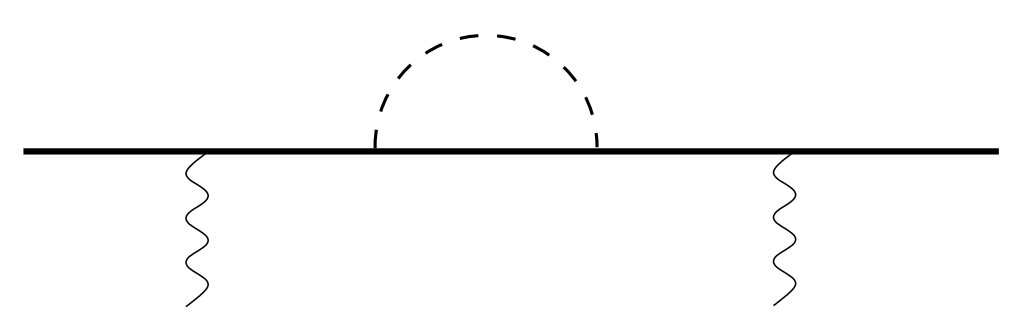

(3)

Fig.1 


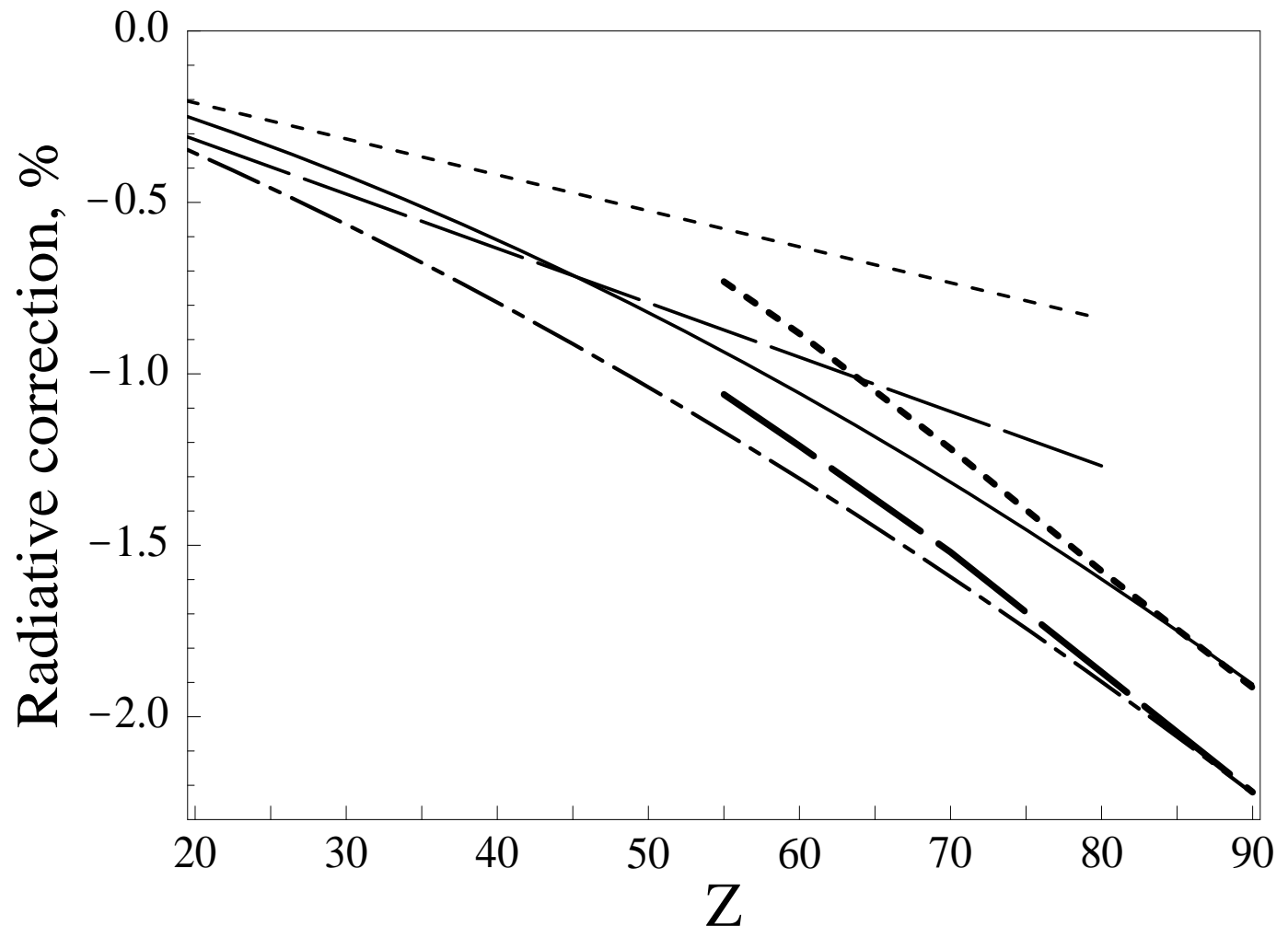

Fig. 2 